\title{
Endoscopia gastroduodenal após administração de nimesulida, monofenilbutazona e meloxicam em cães
}

[Gastroduodenal endoscopy after nimesulide, monophenylbutazone and meloxicam administration in dogs]

\author{
P.R.S. Costa ${ }^{1}$, R.B. Araújo $^{2}$, M.C. Costa ${ }^{1}$, R.E.N. Maia ${ }^{1}$ \\ ${ }^{1}$ Departamento de Veterinária - UFV \\ Av. P.H. Rolfs, s/n \\ 36570-000 - Viçosa, MG \\ ${ }^{2}$ Escola de Veterinária - UFMG - Belo Horizonte, MG
}

\begin{abstract}
RESUMO
Avaliaram-se os aspectos da mucosa gastroduodenal em cães tratados experimentalmente com nimesulida, monofenilbutazona e meloxicam. Foram formados quatro grupos com oito cães. Os grupos 1 , 2 e 3 receberam, respectivamente, tratamento com nimesulida, monofenilbutazona e meloxicam durante 21 dias, e o grupo 4 foi utilizado como controle. Todos os animais foram avaliados por exames endoscópicos do estômago e duodeno antes do experimento e aos 10 e 21 dias de tratamento. Os cães não manifestaram qualquer alteração clínica ou laboratorial durante o período de estudo. A avaliação endoscópica da mucosa gastroduodenal apresentou apenas lesões consideradas de baixo grau. Esses antiinflamatórios mostraram-se seguros para o trato gastrintestinal de cães clinicamente saudáveis.
\end{abstract}

Palavras-chave: cão, antiinflamatório não esteróide, endoscopia, nimesulida, monofenilbutazona, meloxicam

\begin{abstract}
The gastroduodenal mucosa in dogs experimentally treated with nimesulide, monophenylbutazone and meloxicam was evaluated. There were four groups with eight dogs in each. Groups one, two and three were given nimesulide, monophenylbutazone and meloxicam, respectively, during 21 days and group four was used as control. All animals were evaluated by gastroduodenoscopy before the study and on the $10^{\text {th }}$ and $21^{\text {st }}$ days. The dogs did not show any clinical or laboratorial changes during the study. The endoscopic evaluation of gastroduodenal mucosa showed only low degree lesions. These antiinflammatory drugs showed to be safe for the gastrointestinal tract in healthy dogs.
\end{abstract}

Keywords: Nonsteroidal anti-inflammatory drugs, endoscopy, nimesulide, monophenylbutazone, meloxicam

\section{INTRODUÇÃO}

As manifestações clínicas relacionadas ao sistema digestório são freqüentes na clínica de pequenos animais, e uma das principais causas dessa situação está relacionada ao uso indiscriminado de antiinflamatórios não esteróides (AINE) e sua reconhecida ação deletéria sobre a mucosa do trato gastrentérico (Willard, 2003). Essa sensibilidade do trato gastrintestinal aos AINE é atribuída principalmente à inibição da enzima cicloxigenase $(\mathrm{COX})$ e à diminuição na produção de prostaglandinas. As prostaglandinas são responsáveis pela proteção da mucosa gastrintestinal, como a produção de muco e secreção de bicarbonato, a alta taxa de renovação epitelial e o aporte sangüíneo adequado para a mucosa (Kore, 1990; Taylor, 1999; Webb e Twedt, 2003).

Recebido em 13 de julho de 2006

Aceito em 15 de maio de 2007

E-mail: prenato@ufv.br 
Existem duas isoenzimas da cicloxigenase, a cicloxigenase 1 (COX-1) e a cicloxigenase 2 (COX-2). A primeira está normalmente presente nos tecidos, e a segunda é induzida principalmente nos processos inflamatórios. Nos últimos anos, houve grande avanço na terapia antiinflamatória com o desenvolvimento de substâncias com ação seletiva sobre a COX-2. Essas substâncias bloqueiam a síntese dos mediadores da inflamação com mínima ação sobre a COX-1, importante para manutenção dos mecanismos de defesa mediados pelas prostaglandinas (Mathews, 2002; Webb e Twedt, 2003).

A nimesulida é um fármaco antiinflamatório não esteróide que pertence à classe das sulfonanilidas e apresenta ação relativamente seletiva pela COX-2 canina (Ricketts et al., 1998; Toutain et al., 2001). Este é um fármaco pouco estudado em medicina veterinária e não há estudos com acompanhamento endoscópico em relação aos efeitos adversos sobre o trato gastrintestinal de cães. Em pacientes humanos, a nimesulida mostrou menores índices de lesão gastrintestinal do que antiinflamatórios considerados não seletivos (Shah et al., 2001). É possível que a nimesulida possa apresentar boa tolerabilidade gastrintestinal, mostrando-se uma opção segura para o tratamento antiinflamatório na espécie canina.

A monofenilbutazona é um AINE derivado da fenilbutazona. Essa nova formulação apresenta melhor tolerabilidade para a mucosa gastrintestinal do que a fenilbutazona (Loew et al., 1985). Não foram encontrados estudos que avaliaram as alterações na mucosa gastroduodenal pelo uso desse fármaco em cães.

O meloxicam é um AINE com seletividade maior sobre a COX-2 do que a COX-1 e tem se mostrado mais seguro em relação aos efeitos adversos quando comparado aos antiinflamatórios mais antigos, sem ação seletiva sobre a COX-2 (Taylor, 1999; Mathews, 2002).

O estudo teve os objetivos de caracterizar e comparar as alterações endoscópicas da mucosa gastroduodenal de cães clinicamente sadios submetidos a tratamento experimental com nimesulida, monofenilbutazona e meloxicam.

\section{MATERIAL E MÉTODOS}

Incluíram-se 32 cães adultos, sem raça definida, 16 fêmeas e 16 machos, com peso corpóreo entre 10 e $20 \mathrm{~kg}$, clinicamente saudáveis. Todos os animais receberam vermicida ${ }^{1}$, ectoparasiticida ${ }^{2}$ e ficaram em observação (quarentena) durante 15 dias. Os animais foram mantidos no mesmo ambiente, onde receberam a mesma ração comercial para cães adultos ${ }^{3}$ e água à vontade.

Os animais foram separados, por sorteio, em quatro grupos de oito cães, respeitando-se a distribuição equivalente de machos e fêmeas entre os grupos. Os do grupo 1 receberam nimesulida ${ }^{4}$ na dose de $5 \mathrm{mg} / \mathrm{kg}$, uma vez ao dia, pela via oral, conforme citam Toutain et al. (2001), e $1 \mathrm{ml}$ de solução fisiológica, uma vez ao dia, pela via intravenosa; os do grupo 2 receberam cápsulas de placebo ${ }^{5}$, uma vez ao dia, pela via oral, e monofenilbutazona ${ }^{6}$, na dose de $30 \mathrm{mg} / \mathrm{kg}$, uma vez ao dia, pela via intravenosa; os do grupo 3 receberam meloxicam ${ }^{7}$ na dose de $0,1 \mathrm{mg} / \mathrm{kg}$, uma vez ao dia, pela via oral, e $1 \mathrm{ml} \mathrm{de}$ solução fisiológica, uma vez ao dia, pela via intravenosa; e os do grupo 4 receberam cápsulas de placebo $^{5}$, uma vez ao dia, pela via oral, e $1 \mathrm{ml}$ de solução fisiológica, uma vez ao dia, pela via intravenosa. Os tratamentos foram realizados durante 21 dias. Para administração, os comprimidos ou cápsulas foram envoltos em uma porção de ração em pasta ${ }^{7}$.

Durante o período de tratamento, os animais foram avaliados diariamente por meio de exame físico e observação quanto à ingestão de alimento, água e ocorrência de vômitos e/ou diarréia ou qualquer outra alteração clínica digna de nota. Os exames laboratoriais (hemograma completo, urinálise, flotação fecal, uréia, creatinina, alanina aminotransferase e fosfatase alcalina) foram realizados na fase inicial do estudo com o objetivo de selecionar animais sem alterações hematológicas e bioquímicas sugestivas de doença. Esses mesmos exames foram repetidos em todos os animais ao término do período de experimentação.

\footnotetext{
${ }^{1}$ Drontal Cães - Bayer S.A. - São Paulo, Brasil

${ }^{2}$ Top-line - Merial Brasil - Campinas, Brasil

${ }^{3}$ Kanina - Purina - São Paulo, Brasil

${ }^{4}$ Sulidene - Virbac - São Paulo, Brasil

${ }^{5}$ Ração em pasta - Pedigree - São Paulo, Brasil

${ }^{6}$ Monofenew - Vetnil - São Paulo, SP

${ }^{7}$ Maxicam - Ouro Fino - Ribeirão Preto, SP
} 
Todos os animais foram submetidos a exames endoscópicos do estômago e duodeno em três momentos do estudo: antes, aos 10 e aos 21 dias do tratamento. Para realização das endoscopias, os animais foram submetidos a jejum 12 horas antes do exame. Os animais receberam medicação pré-anestésica com acepromazina ${ }^{8}$, pela via intravenosa, e a anestesia geral foi induzida e mantida com tiopental sódico ${ }^{9}$, pela via intravenosa. Foi utilizado um videoendoscópio eletrônico ${ }^{10}$ de $1 \mathrm{~m}$ de comprimento por $9,8 \mathrm{~mm}$ de diâmetro. Com o animal sob anestesia geral, em decúbito lateral esquerdo, deu-se início ao exame com a passagem do endoscópio pelo esfíncter esofágico cranial sob visualização direta. Inicialmente observou-se o trajeto esofágico até a cárdia, e na seqüência, o corpo gástrico, o antro, o piloro e o duodeno. Após, o endoscópio foi retraído para o estômago e feita a manobra de retroversão para exame da cárdia e do fundo gástrico. Todas as observações foram anotadas e registradas em figuras gravadas em computador. Os exames foram feitos sempre pelo mesmo médico veterinário.

Os resultados das observações endoscópicas foram classificados pelo sistema Lanza modificado, conforme citam Shah et al. (2001). Nesse sistema, foram atribuídos escores de zero a quatro conforme a gravidade das lesões na mucosa. O escore zero foi empregado para indicar ausência de lesões. Lesões erosivas ou hemorrágicas únicas receberam escore 1; duas a 10 erosões ou hemorragias puntiformes receberam escore 2; mais de 10 erosões ou hemorragias puntiformes, escore 3; e uma ou mais úlceras de qualquer tamanho, escore 4 . Os escores foram atribuídos separadamente para estômago e duodeno.

As análises dos dados coletados foram feitas, primeiramente, por meio de um histograma, com o objetivo de representar as freqüências dos escores em cada tempo de avaliação para os quatro grupos estudados. Depois, aplicou-se o teste não paramétrico dos sinais, para verificar a mudança dos escores aos 10 e 21 dias em relação ao tempo zero para cada grupo separadamente.

\footnotetext{
${ }^{8}$ Acepran $1 \%$ - Univet S/A Indústria Veterinária - São Paulo, Brasil

${ }^{9}$ Thiopentax - Cristália - Belo Horizonte, Brasil

${ }^{10}$ Videoendoscópio eletrônico Olympus CV-1 - EUA
}

Por fim, foi aplicado o teste não paramétrico de qui-quadrado, para comparar as freqüências de aumento dos escores entre os quatro grupos aos 10 e 21 dias. Todas as análises estatísticas foram feitas no Excel, a 5\% de significância.

\section{RESULTADOS}

Todos os animais mantiveram-se clinicamente bem, sem sinais de anorexia, vômito, diarréia ou perda de peso durante o período de estudo. Os exames laboratoriais realizados antes e após os tratamentos apresentaram resultados dentro dos valores de referência para a espécie canina, conforme citaram Tilley e Smith (2000). Ao exame endoscópico, as lesões foram consideradas de baixa gravidade e receberam escore 1 ou 2 (Fig. 1). Essas lesões, caracterizadas por pontos hemorrágicos únicos ou múltiplos, localizados na região do antro e piloro, foram encontradas nos animais de todos os grupos e nos diferentes momentos da avaliação endoscópica. Com relação à intensidade das lesões, não houve diferenças significativas entre os quatro grupos e entre os momentos antes, durante e após os tratamentos $(\mathrm{P}>0,05)$ (Tab. 1). As regiões do corpo, fundo e cárdia gástrico não apresentaram alterações em nenhum momento do estudo, assim como o duodeno.

As maiores freqüências são do escore 1, independentemente do tempo e do grupo. Essa afirmação poderá ser melhor interpretada com base nos testes não paramétricos aplicados.

Não houve aumento dos escores observados aos 10 e 21 dias em relação ao tempo zero, para todos os grupos $(\mathrm{P}>0,05)$. Todos os grupos apresentaram o mesmo potencial $(\mathrm{P}>0,05)$ de aumentar o valor do escore, com 10 ou 21 dias de tratamento.

\section{DISCUSSÃO}

Não foram observadas diferenças significativas entre os grupos em relação à gravidade das lesões na mucosa gástrica e duodenal antes e depois do tratamento com os AINE. Entre os animais que apresentaram lesões, o grau de intensidade foi considerado leve, sem aparecimento de lesões graves, como úlceras e/ou hemorragia difusa durante os tratamentos. Esses resultados podem estar associados à segurança dos AINE nimesulida, monofenilbutazona e meloxicam para o trato gastrintestinal na espécie canina. 


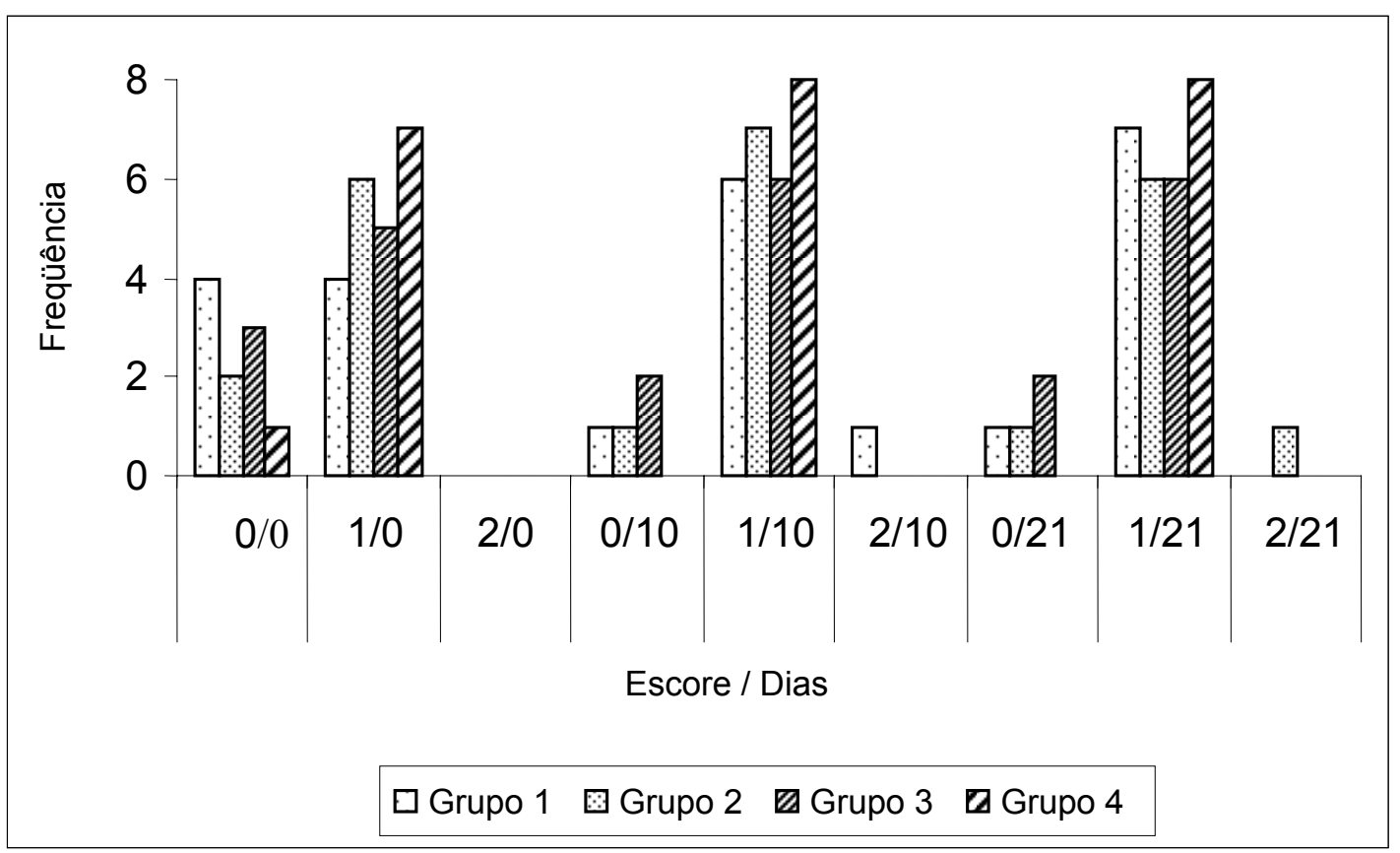

Escore 1: ausência de lesões; escore 2: 1 a10 erosões ou hemorragias puntiformes; escore 3: >10 erosões ou hemorragias puntiformes; escore 4: uma ou mais úlceras. Grupo 1: nimesulida + sol. fisiológica; grupo 2: placebo + monofenilbutazona; grupo 3: meloxicam + sol. fisiológica; grupo 4: placebo + sol. físiológica.

Figura 1. Freqüência do escore de lesões na mucosa gastroduodenal de cães, de acordo com o tempo de avaliação e os grupos.

Tabela 1. Aumento (+) ou manutenção (-) dos escores ${ }^{1}$ da gravidade das lesões na mucosa gastroduodenal de cães, de acordo com os grupos e os momentos de avaliação (dias) e estimativa da probabilidade de significância (valor de P)

\begin{tabular}{|c|c|c|c|c|c|c|c|}
\hline \multicolumn{2}{|c|}{ Grupo 1} & \multicolumn{2}{|c|}{ Grupo 2} & \multicolumn{2}{|c|}{ Grupo 3} & \multicolumn{2}{|c|}{ Grupo 4} \\
\hline 10 & 21 & 10 & 21 & 10 & 21 & 10 & 21 \\
\hline- & - & - & + & - & - & - & - \\
\hline+ & + & - & - & - & - & - & - \\
\hline+ & + & - & - & - & - & + & + \\
\hline- & - & - & - & - & - & - & - \\
\hline- & - & - & - & - & - & - & - \\
\hline- & - & + & + & + & + & - & - \\
\hline+ & + & - & - & - & - & - & - \\
\hline- & - & - & - & - & - & - & - \\
\hline 0,8555 & 0,8555 & 0,9961 & 0,9648 & 0,9961 & 0,9961 & 0,9961 & 0,9961 \\
\hline
\end{tabular}

1: Alteração do escore em relação ao tempo zero. Grupo 1: nimesulida; grupo 2: monofernilbutozona; grupo 3: meloxicam; grupo 4: controle.

A ação seletiva desses AINE pela COX-2 canina pode ter contribuído para reduzir a intensidade das lesões na mucosa. A nimesulida e o meloxicam são considerados AINE com ação seletiva pela COX-2 (Ricketts et al., 1998; Toutain et al., 2001; Mathews, 2002; Wallace, 2003). Em relação à monofenilbutazona, não há estudos detalhados sobre seu modo de ação, porém, pelos resultados obtidos, é possível que esse fármaco também apresente ação predominante sobre a COX-2 e, conseqüentemente, seja mais seguro para a mucosa gastrentérica do que os AINE sem ação seletiva. Os AINES com ação seletiva pela COX2 são considerados mais seguros para o trato gastrintestinal (Forsyth et al., 1998; Reimer et 
al., 1999; Mathews, 2002). Eles apresentam mínima ação sobre a COX-1, considerada a principal responsável pela manutenção dos mecanismos de defesa da mucosa.

Os resultados obtidos assemelham-se aos de Forsyth et al. (1998) e Reimer et al. (1999), que descreveram os achados clínicos e endoscópicos de cães clinicamente saudáveis submetidos a tratamento com AINES durante 28 dias. Eles trabalharam com os antiinflamatórios carprofeno, etodolac e meloxicam, todos considerados com ação predominantemente sobre a COX-2, e não verificaram diferenças significativas entre os fármacos. Mesmo após 28 dias de tratamento, os cães apresentavam-se assintomáticos e com lesões endoscópicas de grau leve, como pontos hemorrágicos e erosões focais. Essas lesões de baixa intensidade também foram constatadas no presente estudo (Fig. 2).

O emprego de cães clinicamente saudáveis pode ser outro fator que ajuda a explicar a baixa intensidade de lesões na mucosa gastroduodenal, além da segurança dos antiinflamatórios empregados. Indivíduos hígidos apresentam os mecanismos de proteção da mucosa preservados, ao contrário do animal politraumatizado ou em estado de choque ou portador de alguma doença crônica. Nessas situações debilitantes, a mucosa torna-se mais susceptível a injúrias devido à maior intensidade do estresse, diminuição do aporte sangüíneo para a mucosa e à conseqüente perda dos mecanismos de proteção da mucosa, como a produção de muco e bicarbonato, a capacidade de renovação epitelial e a manutenção do próprio fluxo sangüíneo (Guilford e Strombeck, 1996).

Lesões hemorrágicas puntiformes e erosivas focais foram encontradas em animais de todos os grupos. A causa dessas lesões pode estar associada ao estresse nos indivíduos do grupocontrole. Nos grupos tratados, os AINES e o estresse podem ter contribuído, em conjunto, para o desencadeamento das lesões. Alterações semelhantes foram vistas por Forsyth et al. (1998) no estômago de cães clinicamente sadios, quando o estresse foi apontado como uma das possíveis causas.
O confinamento e a manipulação diária dos animais para administração dos tratamentos contribuíram para intensificar o estresse durante o estudo. A fisiopatologia das lesões por estresse na mucosa gástrica é controversa. O principal fator incriminado é a redução do fluxo sangüíneo na mucosa por vasoconstrição esplâncnica, associada ao aumento do tônus simpático e a níveis elevados de catecolaminas circulantes. A vasoconstrição não ocorre de maneira uniforme em toda a mucosa, portanto produz áreas focais de isquemia, onde ocorre quebra da barreira de muco e bicarbonato, penetração de ácido da luz para a mucosa e desenvolvimento de lesão erosiva, que pode evoluir para a formação de úlcera. Lesões de reperfusão por radicais livres no momento em que a circulação é restabelecida em um foco previamente isquêmico também podem contribuir para a gênese das alterações (Guilford e Strombeck, 1996).

A incidência de lesões foi mais freqüente no antro gástrico e próximo ao piloro. Segundo Guilford e Strombeck (1996), a mucosa do antro é mais susceptível a lesões isquêmicas pela menor quantidade de interconexões entre os capilares que a mucosa do corpo e fundo gástrico. Dessa forma, uma lesão endotelial e oclusão de um capilar nessa região resultaria em uma área isquêmica maior, levando ao aparecimento de pontos hemorrágicos ou erosões e úlceras focais nos casos mais avançados.

Os AINES são associados com vários efeitos adversos. $O$ trato gastrintestinal é o mais freqüentemente acometido. Em segundo lugar, aparecem o sistema urinário e as discrasias sangüíneas. No presente estudo, não foram observadas alterações em outros sistemas. Os achados de exame físico, hemograma e testes bioquímicos realizados antes e após os tratamentos não apresentaram diferenças dignas de nota. Estes resultados assemelham-se aos de Forsyth et al. (1998) e Reimer et al. (1999), que não observaram alterações clínicas e laboratoriais antes e após o tratamento de 28 dias com carprofeno, etodolac e meloxicam em cães clinicamente saudáveis. 


\section{Costa et al.}
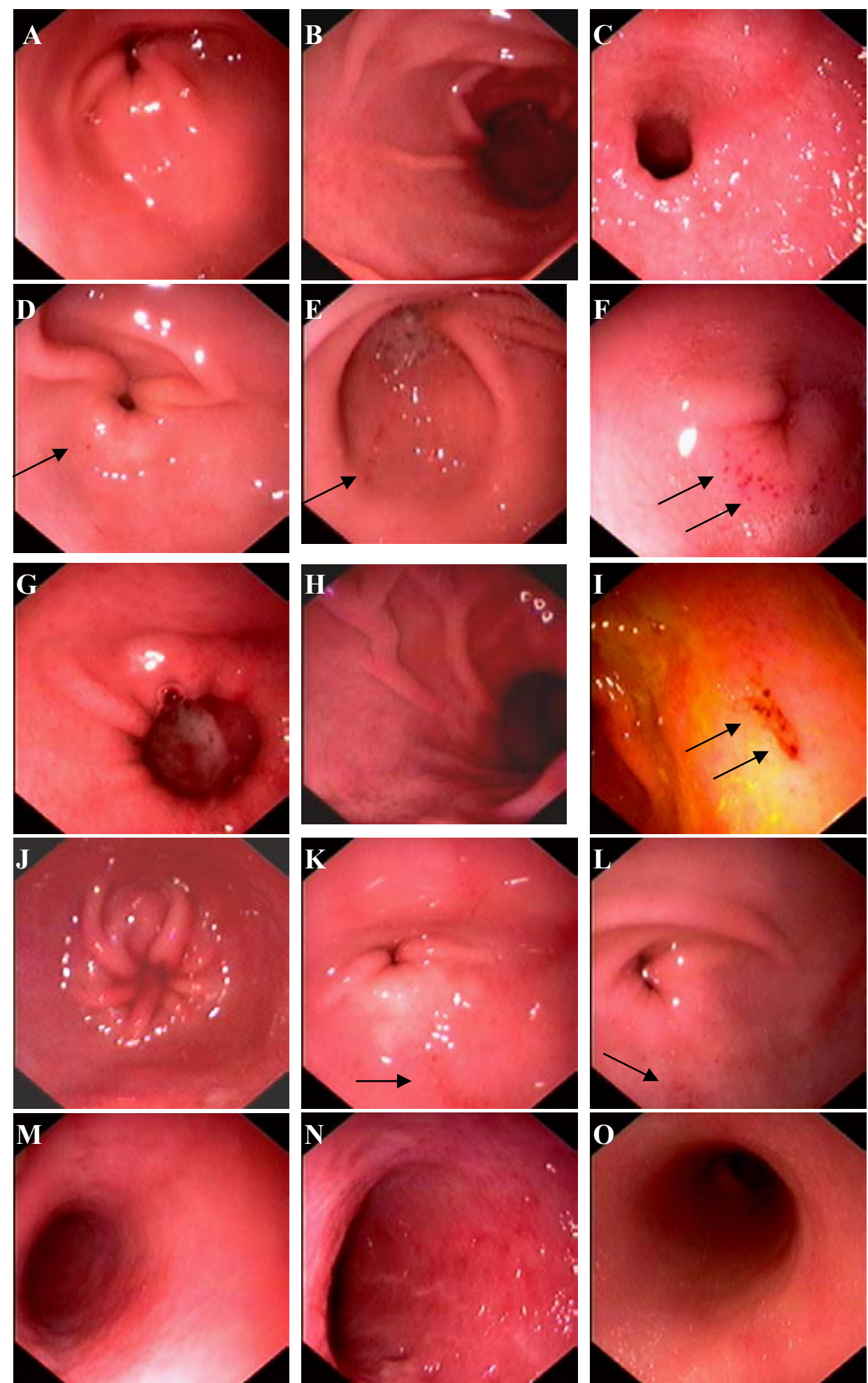

Figura 2. Imagens endoscópicas. A, B, e C- grupo 1, momentos antes, 10 e 21 dias, respectivamente. Mucosa do antro e piloro sem alterações. D, E e F-grupo 2, momentos antes, 10 e 21 dias, respectivamente. Mucosa do antro e piloro com pontos hemorrágicos (setas). G, H e I- grupo 3, momentos antes, 10 e 21 dias, respectivamente. Mucosa do antro e piloro sem alterações (G,H) e erosões localizadas (I), (setas). J, K e L- grupo 4, momentos antes, 10 e 21 dias, respectivamente. Mucosa do antro e piloro sem alterações (J) e com pontos hemorrágicos localizados (K,L), (setas). M, N e O- Mucosa duodenal sem alterações aos 21 dias de tratamento dos grupos 1,2 , e 3 , respectivamente. 


\section{CONCLUSÃO}

Os antiinflamatórios nimesulida, monofenilbutazona e meloxicam são seguros para a mucosa gastroduodenal de cães clinicamente saudáveis.

\section{REFERÊNCIAS BIBLIOGRÁFICAS}

FORSYTH, S.F.; GUILFORD, W.G.; HASLETT, S.J. et al. Endoscopy of the gastroduodenal mucosa after carprofen, meloxicam and ketoprofen administration in dogs. J. Small Anim. Pract. v.39, p.421-424, 1998.

GUILFORD, W.G.; STROMBECK, D.R. Acute gastritis. In: GUILFORD, W.G.; CENTER, S.A.; STROMBECK, D.R. et al. Small animal gastroenterology. 3.ed. Philadelphia: Saunders, 1996. p.261-274.

KORE, A.M. Toxicology of nonsteroidal antiinflamatory drugs. Vet. Clin. N. Am.: Small Anim. Pract., v.20, p.419-430, 1990.

LOEW, D.; SCHUSTER, O.; KNOELL, H.E. et al. Pharmacology, toxicology and pharmacokinetics of mofebutazone. ZRheumatol., v.44, p.186-192, 1985.

MATHEWS, K.A. Non-steroidal antiinflammatory analgesics: a review of current practice. J. Vet. Emerg. Crit. Care, v.12, p.89-97, 2002.

REIMER, M.E.; JOHNSTON, S.A.; LEIB, M.S. et al. The gastroduodenal effects of buffered aspirin, carprofen, and etodolac in healthy dogs. J. Vet. Intern. Med. v.13, p.472-477, 1999.
RICKETTS, A.P.; LUNDY, K.M.; SEIBEL, S.B. Evaluation of selective inhibition of canine cyclooxygenase 1 and 2 by carprofen and other nonsteroidal anti-inflammatory drugs. Am. J. Vet. Res., v.59, p.1441-1446, 1998.

SHAH, A.A.; THJODLEIFSSON, B.; MURRAY, F.E. et al. Selective inhibition of COX-2 in humans is associated with less gastrointestinal injury: a comparison of nimesulide and naproxen. Gut, v.48, p.339-346, 2001.

TAYLOR, P.M. Newer analgesics: Nonsteroid anti-inflammatory drugs, opioids, and combinations. Vet. Clin. N. Am.: Small Anim. Pract., v.29, p.719-735, 1999.

TILLEY, L.P.; SMITH, F.W.K. The 5-minute veterinary consult. 2.ed. Philadelphia: Lippincott Williams \& Wilkins, 2000. p.1323-1325.

TOUTAIN, P.L.; CESTER, C.C.; HAAK, T. et al. A Pharmacokinetic/pharmacodynamic approach vs. a dose titration for the determination of a dosage regimen: the case of nimesulide, a Cox-2 selective nonsteroidal antiinflammatory drug in the dog. J. Vet. Pharmacol. Therap., v.24, p.43-55, 2001.

WALLACE, J.M. Meloxicam. Comp.Cont. Educ. Pract. Vet., v.25, p.64-65, 2003.

WEBB, C.; TWEDT, D.C. Canine gastritis. Vet. Clin. N. Am.: Small Anim. Pract., v.33, p.969985, 2003.

WILLARD, M.D. Disorders of the stomach. In: NELSON, R.W.; COUTO, C.G. Small animal internal medicine. 3.ed. St. Louis: Mosby, 2003. cap.32, p.418-430. 